\title{
Sono e Comportamento em Crianças Atendidas em um Serviço de Psicologia
}

\author{
Renatha El Rafihi-Ferreira' \\ Edwiges Ferreira de Mattos Silvares \\ Universidade de São Paulo - SP - Brasil \\ Maria Laura Nogueira Pires \\ Universidade Estadual Paulista "Júlio de Mesquita Filho" - SP - Brasil \\ Francisco Baptista Assumpção Junior \\ Universidade de São Paulo, São Paulo - SP - Brasil \\ Cynthia Borges de Moura \\ Universidade Estadual do Oeste do Paraná - PR - Brasil
}

\begin{abstract}
Resumo: Problemas de sono são frequentes na infância e podem causar prejuízos à criança. Há uma associação possível entre distúrbios de sono infantil e problemas de comportamento. O objetivo do presente estudo foi avaliar as relações entre problemas internalizantes e externalizantes e problemas de sono em crianças da faixa pré-escolar, avaliadas por suas mães pelo Child Behavior Checklist (CBCLI 1 $/ 2-5$ anos), quando estas buscaram atendimento em uma clínica-escola de psicologia. Participaram do estudo 83 mães de crianças (sendo seus filhos: 58 meninos e 25 meninas) cujas idades variaram entre 2 e 5 anos. Análises indicaram que $29 \%$ das crianças apresentavam problemas com o sono, entre os quais, os mais frequentes foram "não querer dormir sozinho" e "resistir a ir para a cama". Os dados sugeriram ainda uma associação entre problemas de sono e comportamentos internalizantes $(R P=1,4 ; p<0,05)$ e externalizantes $(R P=I, 4 ; p<0,05)$.
\end{abstract}

Palavras-chave: insônia; comportamento externalizante; comportamento internalizante; criança; Child Behavior Checklist.

\section{SLEEP AND BEHAVIOR IN CHILDREN ASSISTED IN A PSYCHOLOGY SERVICE}

\begin{abstract}
Sleep problems are common in childhood and can cause damage in children. There is a possible association between childhood sleep disorders and behavioral problems. The aim of this study was to evaluate the relationship between internalizing and externalizing problems and sleep problems in children of pre-school age, assessed by their mothers through the Child Behavior Checklist ( CBCLI $1 / 2-5$ years) when seeking care at a clinic school psychology. The study included 83 mothers of children (and their children were: 58 boys and 25 girls) whose ages ranged between 2 and 5 years. Analysis indicated that $29 \%$ of children had sleep problems among which the most frequent were "not wanting to sleep alone" and "resist the bed". The data also suggested an association between sleep problems and internalizing $(P R=1,4 ; p<0,05)$ and externalizing $(P R=I, 4 ; p<0,05)$ behaviors.
\end{abstract}

Keywords: insomnia; externalizing behavior; internalizing behavior; children; Child Behavior Checklist.

1 Endereço para correspondência: Renatha El Rafihi-Ferreira, Universidade de São Paulo, Instituto de Psicologia, Avenida Professor Mello Moraes, 1721, Butantã - São Paulo - Brasil. CEP: 05508-030. E-mail: rerafihi@usp.br. 
Renatha El Rafihi-Ferreira, Edwiges Ferreira de Mattos Silvares, Maria Laura Nogueira Pires, Francisco Baptista Assumpção Junior, Cynthia Borges de Moura

\title{
EL SUEÑO Y EL COMPORTAMIENTO EN LOS NIÑOS ATENDIDOS EN UN SERVICIO DE PSICOLOGÍA
}

\begin{abstract}
Resumen: Los problemas del sueño son comunes en la infancia y pueden causar daños en los niños. Existe una posible asociación entre los trastornos del sueño de la infancia y los problemas de comportamiento. El objetivo de este estudio fue evaluar la relación entre los problemas de internalización y externalización y los trastornos del sueño en niños en edad preescolar, evaluados por sus madres por medio del Child Behavior Checklist (CBCLI 1 1 2 -5 años) cuando buscaban tratamiento en una clínica-escuela de psicología. El estudio incluyó a 83 madres y sus hijos (y sus niños: 58 niños y 25 niñas), con edades comprendidas entre los 2 y 5 años. El análisis indicó que $29 \%$ de los hijos tenían problemas de sueño entre los cuales los más frecuentes fueron "no querer dormir solo" y "resistir a la cama". Los datos también sugieren una asociación entre los trastornos del sueño y comportamientos de internalización $(P R=1,4 ; p<0,05)$ y de externalización $(P R=1,4 ; \mathrm{P}<0,05)$.
\end{abstract}

Palabras clave: insomnio; comportamiento de exteriorización; comportamiento de internalización; niños; Child Behavior Checklist.

Distúrbios de sono são frequentes na população infantil, sendo que o problema de sono mais comum entre as crianças é a insônia comportamental (Hall, Zubrick, Silburn, Parsons, \& Kurinczuk, 2007). De acordo com os critérios especificados pela Classificação Internacional de Distúrbios de Sono (American Academy of Sleep Medicine, 2005), a insônia comportamental infantil se manifesta como dificuldade para adormecer quando é colocada na cama ou de permanecer dormindo ao longo da noite, despertando várias vezes, resistindo a voltar para a cama e encontrando dificuldade para adormecer sem a presença dos pais. Há três tipos de insônia comportamental na infância (American Academy of Sleep Medicine, 2005; Owens \& Mindell, 2011): 1. insônia de associação para iniciar o sono; 2 . insônia por dificuldades de imposição de limites; ou 3. a combinação entre elas.

A insônia de associação geralmente se manifesta com despertares noturnos frequentes, sendo comumente resultado de associações inapropriadas com o sono. Seu início se associa a fatores externos como colo, mamadeira, televisão, música e presença dos pais. Crianças que adormecem com contato físico ou envolvimento parental ativo têm maior probabilidade de precisarem de ajuda para voltarem a dormir depois dos despertares que normalmente acontecem durante a noite. Já as crianças com insônia comportamental do tipo dificuldades de imposição de limites relutam, de forma característica, na hora de ir para a cama ou atrasam esse momento com repetidas requisições (mais uma história, um beijo a mais etc.) (Owens \& Mindell, 2011).

A insônia comportamental infantil é tipicamente observada em crianças de 0 a 5 anos, embora possa persistir na idade escolar e na adolescência, especialmente em crianças com necessidades especiais (Owens \& Mindell, 2011). De forma geral, sua prevalência na população infantil varia entre $20 \%$ e 30\% (Mindell, Kuhn, Lewin, Meltzer, $\&$ Sadeh, 2006). No entanto, os problemas de sono variam conforme a idade. A dificuldade para iniciar o sono e o despertar noturno ocorrem em $40 \%$ dos bebês recém-nascidos e em $20 \%$ a $50 \%$ dos pré-escolares; já a resistência ao dormir é relatada em $15 \%$ a $27 \%$ das crianças em idade escolar (Owens \& Mindell, 2011). Resultados de es- 
tudo nacional (Pires, Vilela, \& Câmara, 2012) apontam que uma parcela importante de crianças em idade pré-escolar resiste a ir para a cama (56\%), tem dificuldade de adormecer $(30 \%)$, antes de adormecer fica agitada, nervosa ou com medo (22\%), acorda várias vezes durante a noite (35\%) e fica sonolenta durante o dia (35\%).

Como o sono é um processo fisiológico e comportamental fundamental para saúde e bem-estar do organismo, os problemas de sono em crianças são fonte de preocupação constante. Os distúrbios de sono na infância podem causar prejuízos nas crianças e em seus familiares (Hall et al., 2007). Estudiosos (Gregory \& Sadeh, 2012; Tikotzky \& Sadeh, 2010) apontam que a má qualidade de sono na infância pode prejudicar a funcionalidade diurna e afetar aspectos cognitivos, comportamentais, emocionais e acadêmicos da criança.

Existem vários estudos nos quais os pesquisadores apontam uma possível associação entre distúrbios de sono e problemas de comportamento, avaliados pelo Child Behavior Checklist (CBCL). Problemas de comportamento podem ocorrer de forma internalizante ou externalizante. Os comportamentos internalizantes são os que se expressam em relação ao próprio indivíduo. Por essa razão, problemas no perfil internalizante são mais difíceis de serem detectados pelos cuidadores. Tais comportamentos podem ser identificados quando existem preocupação em excesso, retraimento, tristeza, timidez, insegurança e medos, e estão geralmente presentes na depressão, no isolamento social e na ansiedade (Achenbach \& Rescorla, 2000). Já os comportamentos referentes ao perfil externalizante são ações que se voltam para o ambiente e para outras pessoas. Por essa característica, os problemas externalizantes podem ser mais facilmente identificados por observadores externos, como pais, professores ou cuidadores (Achenbach \& Rescorla, 2000). Esses comportamentos envolvem agressão, agitação e impulsividade.

O estudo de Stein, Mendelsohn, Obermeyer, Amromin, \& Benca (2001) avaliou a prevalência de problemas de sono em uma população não clínica de crianças escolares e investigou se problemas de sono foram associados a problemas de comportamento. Conforme os autores, problemas internalizantes e externalizantes apresentam correlações com parassonia, insônia, sono ruidoso e cansaço diurno. A pesquisa de Shang, Gau, \& Soong (2006) com 1391 crianças escolares demonstrou que crianças com problemas de sono apresentaram pontuação maior nas oito síndromes comportamentais derivadas do $\mathrm{CBCL}$ do que crianças sem problemas de sono. Os problemas relacionados ao sono foram positivamente relacionados a escores internalizantes e externalizantes no estudo de Storch, Murphy, Lack, Geffken, Jacob e Goodman (2008) com crianças e adolescentes de 8 a 17 anos que apresentavam transtorno obsessivo compulsivo.

Investigações sobre associações entre problemas de comportamento e de sono também foram realizadas com crianças em idade pré-escolar. O estudo de Sourander (2001) com 525 crianças com 3 anos, recrutadas em serviços pediátricos, avaliou problemas de sono e de comportamento e por meio do $\mathrm{CBCL}$, e os resultados demonstraram que os problemas de sono foram associados a dormir com os pais na mesma cama ou compartilhando o quarto. Dormir no mesmo quarto que os pais foi associado a 
escores altos no perfil externalizante. Todavia, a associação entre problema de sono e comportamento não foi encontrada na pesquisa de Hiscock, Bayer, Hampton, Ukoumunne e Wake (2008) com crianças de 2 anos que foram recrutadas por meio de serviços pediátricos para tratamento de problemas de sono. Essas crianças não apresentaram problemas de comportamento, isto é, não apresentaram pontuações consideradas clínicas no CBCL.

Um fator preocupante é que os problemas de sono na infância podem persistir. Scher, Zukerman e Epstein (2005) encontraram associações entre dificuldades com o sono no primeiro ano de vida e posteriores problemas de comportamento aos 3 e 4 anos. Ainda neste contexto, Hall et al. (2007) apontaram que escores mais altos de problemas de sono aos 3 anos foram preditores de comportamento agressivo aos 4 anos. Tikotzky e Sadeh (2010) indicam que problemas de sono na infância podem durar até a vida adulta.

Por meio de um estudo longitudinal, Gregory, Ende, Willis e Verhulst (2008) investigaram associações entre problemas de sono durante a infância, identificados por questões do $\mathrm{CBCL}$, e subsequentes dificuldades emocionais e comportamentais, acessadas por meio do instrumento Young Adult Self-Report. Os resultados demonstraram que crianças e adolescentes que dormiam em menor quantidade de horas durante a fase de desenvolvimento, na idade adulta, apresentaram chances maiores de comprometimento nas escalas de ansiedade/depressão e na escala de comportamento agressivo. Os autores apontam que dificuldades relacionadas ao sono na infância podem constituir indicadores de risco de dificuldades cognitivas e comportamentais na vida adulta.

No que diz respeito à faixa etária pré-escolar, ainda, não há um consenso sobre a associação entre sono e comportamento; há poucas pesquisas publicadas com crianças nesta faixa etária. Neste cenário, um estudo recente (Sivertsen, Harvey, Recihborn-Kjennerud, Torgersen, Ystrom, \& Hysing, 2015) indicou que despertares noturnos e curta duração de sono aos 18 meses predizem problemas de comportamento internalizante e externalizante aos 5 anos. Contudo, ao considerar o território nacional, constata-se uma precariedade de estudos publicados que relacionam sono e comportamento.

Tendo em vista a importância do sono para a saúde e a qualidade de vida infantil, justifica-se a necessidade de pesquisas que investiguem associações entre problemas de sono e problemas de comportamento. O objetivo do presente estudo, portanto, foi avaliar as relações entre problemas de sono e comportamentos internalizantes e externalizantes, avaliados pelo CBCL1 $1 / 2-5$ anos, em crianças cujas mães buscaram atendimento psicológico em clínica-escola de psicologia.

\section{Método}

\section{Participantes}

Participaram do estudo 83 duplas mãe-criança que foram recrutadas na lista de espera para atendimento infantil da Clínica Psicológica da Universidade Estadual de Londrina-PR ou que atenderam aos anúncios de divulgação pela mídia (jornais, rádio 
e televisão) convidando pais de crianças em idade pré-escolar com problemas de comportamento a se submeterem a uma avaliação na clínica psicológica com vistas a receberem orientação. A idade das crianças (58 meninos e 25 meninas) variou de 2 a 5 anos, com média de 3,5 e desvio padrão de 1,03. Os dados das crianças, fornecidos pelas mães, foram coletados como parte da avaliação inicial para outra pesquisa, realizada pela última autora, que teve como objetivo caracterizar o perfil da clientela pré-escolar.

\section{Instrumento}

Inventário de Comportamentos para Crianças entre $1 \frac{1}{2}$ a 5 anos (CBCL11/2 a 5 anos) - Trata-se de um instrumento destinado a obter taxas padronizadas de problemas comportamentais de crianças a partir do relato dos pais. A versão pré-escolar foi elaborada por Achenbach e Rescorla (2000) para avaliar questões específicas da faixa etária entre um $11 / 2$ a 5 anos. Esta versão é amplamente utilizada em estudos nacionais e é composta por 99 itens destinados à avaliação dos problemas de comportamento da criança. O informante é orientado a quantificar os comportamentos apresentados pela criança nos últimos dois meses em uma escala de 0 a 2 pontos, que indicam: 0 - item falso ou comportamento ausente; 1 - item parcialmente verdadeiro ou comportamento às vezes presente; 2 - item bastante verdadeiro ou comportamento frequentemente presente. A versão para pais de crianças de 11/2 a 5 anos avalia as seguintes síndromes: Reatividade Emocional, Ansiedade/Depressão, Queixas Somáticas, Problemas de Atenção e Comportamento Agressivo e Problemas de Sono. Por meio da análise dos itens dessas síndromes, obtém-se também uma caracterização da criança quanto a seu Funcionamento Global (clínica, normal ou limítrofe) e nos perfis Internalizante e Externalizante. Problemas internalizantes são compostos pelas seguintes síndromes: Reatividade Emocional, Ansiedade/Depressão, Queixas Somáticas, Retraimento/Depressão. Os problemas externalizantes são compostos pelas síndromes Problemas de Atenção e Comportamento Agressivo. A avaliação do instrumento é computadorizada, realizada por meio de software específico. Os valores para análise dos escores $\mathrm{T}$ em Funcionamento Global, Perfil Internalizante e Perfil Externalizante são: limítrofe - escores entre 60 e 63; clínico - escores iguais ou superiores a 64; e normal - escores inferiores a 60. Para as síndromes, os escores limítrofes são entre 65 e 69; clínico - escores iguais ou superiores a 70; e normal - escores inferiores a 65.

Dados os objetivos da presente pesquisa, a análise se restringiu às subescalas relativas aos Problemas de Sono, Perfil Externalizante e Perfil Internalizante. A subescala Problemas de Sono foi utilizada para avaliação de sono infantil em outros estudos (Gregory et al., 2008; Hall et al., 2007; Hiscock et al,. 2008, Shang et al., 2006; Sourander, 2001) e mostrou correlações positivas com outras medidas de relato parental sobre o sono da criança (Hall, Scher, Zaidman-Zait, Espezel, \& Warnock, 2011).

As sete questões que compõem a categoria Problemas de Sono também foram analisadas separadamente. As questões abordadas neste item são: 1. a criança não quer 
dormir sozinha; 2. problemas para dormir; 3. pesadelos; 4. resiste a ir para a cama; 5. dorme pouco; 6. fala e/ou chora enquanto dorme; e 7. desperta frequentemente.

\section{Procedimentos de coleta de dados}

Os dados foram coletados no Laboratório de Estudos do Comportamento Humano e nas salas de atendimento da Clínica Psicológica da Universidade Estadual de Londrina-PR, por uma pesquisadora experiente na aplicação do instrumento. As mães que se inscreveram para a participação no programa de orientação a pais foram esclarecidas quanto ao uso dos resultados das avaliações iniciais de seu filho. As mães que concordaram, assinaram o Termo de Consentimento Livre e Esclarecido e, individualmente, preencheram o $C B C L$ versão $11 \frac{1}{2}$ a 5 anos, em sessões de aproximadamente 40 minutos. A aplicadora forneceu as explicações sobre o preenchimento do instrumento e questionou a mãe se ela preferia responder com ou sem auxílio. Mesmo no caso em que a mãe optou por responder sozinha, a aplicadora permaneceu na sala para reduzir eventuais dúvidas.

\section{Procedimentos de análise dos dados}

Foram realizadas análises descritivas (proporções, médias e desvio padrão). Os dados dos problemas de sono foram divididos em três grupos de acordo com suas frequências (ausente, ocasional e frequente). As comparações entre grupos foram feitas com Anova de uma via, seguida pelo teste de comparações múltiplas de Duncan, quando apropriado. Medidas de associação entre problemas de sono e problemas internalizantes e externalizantes foram feitas por meio do cálculo da razão de prevalência (RP).

\section{Considerações éticas}

Este estudo recebeu a aprovação do Comitê de Ética em Pesquisa Envolvendo Seres Humanos da Universidade Estadual de Londrina (CAAE n. 0121.0.268.000-07). As mães das crianças foram chamadas para uma devolução dos dados do CBCL. Para as mães das crianças que receberam escore clínico para problemas de comportamento, foram ofertadas duas a três sessões individuais com as terapeutas da equipe de pesquisa para receber orientações quanto aos problemas que enfrentavam com seus filhos. As mães que desejaram dar continuidade aos atendimentos foram encaminhadas para a clínica-escola da Universidade.

\section{Resultados}

Os resultados são apresentados na seguinte ordem: 1. problemas de sono; 2 . problemas de sono e perfil internalizante; 3 . problemas de sono e perfil externalizante.

\section{Problemas de sono}

A Tabela 1 refere-se à proporção de crianças que apresentaram problemas de sono avaliados pelo $\mathrm{CBCL}$. 
Tabela I. Frequência de crianças com problemas de sono avaliados pelo CBCL $(\mathbf{N}=\mathbf{8 3})$

\begin{tabular}{lccc}
\hline Questões de sono $-\mathrm{CBCL}$ & 1 - Ocasional & 2 - Frequente & Total \\
\hline A criança não quer dormir sozinha & $17(21 \%)$ & $45(54 \%)$ & $62(75 \%)$ \\
Problemas para dormir & $23(28 \%)$ & $19(23 \%)$ & $42(51 \%)$ \\
Pesadelos & $25(30 \%)$ & $9(11 \%)$ & $34(41 \%)$ \\
Resiste à cama & $26(31 \%)$ & $29(35 \%)$ & $55(66 \%)$ \\
Dorme pouco & $19(23 \%)$ & $9(11 \%)$ & $28(34 \%)$ \\
Fala e/ou chora enquanto dorme & $26(31 \%)$ & $16(19 \%)$ & $42(51 \%)$ \\
Desperta durante a noite & $24(29 \%)$ & $17(21 \%)$ & $41(50 \%)$ \\
\hline
\end{tabular}

Fonte: Elaborada pelos autores.

Observa-se que todas as queixas de sono apresentadas no $\mathrm{CBCL}$ foram relatadas pelas mães, constatando-se um número considerável de crianças que frequentemente não queriam dormir sozinhas (54\%), resistiam frequentemente a ir para a cama (35\%), apresentavam continuadamente problemas para dormir (23\%) e despertavam repetidas vezes durante a noite (21\%). Em contrapartida, poucas crianças tinham pesadelos frequentes e dormiam pouco $(n=9 ; 11 \%)$.

A partir do ponto de corte estabelecido por Achenbach e Rescorla (2000), os resultados revelaram que $29 \%(n=24)$ das crianças atendiam aos critérios para Problemas de Sono (escores T igual ou maior que 65). A idade média dessas crianças foi de 3,25 anos (DP $=1,07)$ anos, sendo que $54 \%(n=13)$ tinham 2 e 3 anos e $46 \%(n=11)$ entre 4 e 5 anos. Destas 24 crianças, a maioria era do sexo masculino $(n=14,58 \%)$.

Problemas de Sono e Perfil Internalizante

Tabela 2. Comparação entre os escores T médios obtidos no Perfil Internalizante por crianças $(\mathbf{N}=\mathbf{8 3})$ de acordo com a frequência do problema de sono. Dados expressos como média (dp)

\begin{tabular}{lcccccc}
\hline & \multicolumn{4}{c}{ Frequência do problema de sono } & & \\
\cline { 2 - 5 } Tipo de problema de sono & Ausente & Ocasional & Frequente & & $F(2,80)$ & P \\
\hline Não quer dormir & $64,9(9,1)$ & $65,5(6,3)$ & $64,7(9,7)$ & & 0,5 & 0,9 \\
Problema para dormir & $62,6(8,9)$ & $65,0(10,0)$ & $69,8(4,6) *$ & 4,6 & 0,01 \\
Resiste para dormir & $61,0(10,5)$ & $64,8(8,5)$ & $68,8(5,3) \#$ & 6,1 & 0,003 \\
Dorme pouco & $64.0(8.6)$ & $65.5(10.6)$ & $69.2(4.6)$ & & 1,4 & 0,25 \\
\hline
\end{tabular}


Tabela 2. Comparação entre os escores T médios obtidos no Perfil Internalizante por crianças $(\mathbf{N}=\mathbf{8 3})$ de acordo com a frequência do problema de sono. Dados expressos como média (dp) (conclusão)

\begin{tabular}{lcccccc}
\hline & \multicolumn{4}{c}{ Frequência do problema de sono } & & \\
\cline { 2 - 4 } Tipo de problema de sono & Ausente & Ocasional & Frequente & & $F(2,80)$ & \\
\hline Desperta & $63,4(9,2)$ & $63,6(9,1)$ & $70,6(4,7)^{*}$ & & 4,9 & 0,01 \\
Pesadelo & $63,0(9,3)$ & $66,6(8,1)$ & $70,8(4,3) \#$ & & 3,9 & 0,02 \\
Fala, chora & $62,8(10,0)$ & $64,5(7,2)$ & $71,2(4,2) *$ & & 5,9 & 0,004 \\
\hline
\end{tabular}

$*=p<0,05$, difere dos demais grupos, teste de comparações múltiplas de Duncan.

$\#=p<0,05$, difere do grupo Ausente, teste de comparações múltiplas de Duncan.

Fonte: Elaborada pelos autores.

A Tabela 2 demonstra a comparação entre os escores T médios obtidos no Perfil Internalizante por crianças $(\mathrm{N}=83)$ de acordo com a frequência do problema de sono. Constata-se que os escores no perfil internalizante de crianças que possuem problemas frequentes para dormir resistem a ir para a cama, despertam várias vezes durante a noite, têm pesadelos ou falam/choram durante o sono são significativamente mais altos do que os escores encontrados em crianças cujas mães não fizeram referência a essas dificuldades com o sono.

Dos 64 casos internalizantes encontrados ( $77 \%$ da amostra total), 23 (36\%) tinham problemas com o sono. Por outro lado, entre os 19 participantes sem problemas internalizantes, apenas $1(5,3 \%)$ tinha problema com o sono. O cálculo da razão de prevalência demonstrou que crianças com problemas de sono têm chances $40 \%$ maiores de apresentarem problemas internalizantes em comparação com as que não apresentam dificuldades com o sono ( $R P=1,4$; IC $95 \%=1,14-1,67)$.

Problemas de Sono e Perfil Externalizante

Tabela 3. Comparação entre os escores T médios obtidos no Perfil Externalizante por crianças $(\mathbf{N}=\mathbf{8 3})$ de acordo com a frequência do problema de sono. Dados expressos como média (dp)

Frequência do problema de sono

\begin{tabular}{lccccc}
\cline { 2 - 4 } Tipo de problema de sono & Ausente & Ocasional & Frequente & $\mathrm{F}(2,80)$ & $\mathrm{P}$ \\
\hline Não quer dormir & $64,0(8,0)$ & $61,6(13,1)$ & $67,8(11,8)$ & 2,16 & 0,12 \\
Problema para dormir & $61,9(12,1)$ & $67,6(9,5)$ & $71,2(9,4) \#$ & 5,28 & 0,006 \\
Resiste para dormir & $59,7(13,0)$ & $66,8(10,2)$ & $70,2(8,4) *$ & 7,13 & 0,001 \\
\hline
\end{tabular}


Tabela 3. Comparação entre os escores $\mathbf{T}$ médios obtidos no Perfil Externalizante por crianças $(\mathbf{N}=\mathbf{8 3})$ de acordo com a frequência do problema de sono. Dados expressos como média (dp) (conclusão)

\begin{tabular}{|c|c|c|c|c|c|}
\hline \multirow[b]{2}{*}{ Tipo de problema de sono } & \multicolumn{3}{|c|}{ Frequência do problema de sono } & \multirow[b]{2}{*}{$F(2,80)$} & \multirow[b]{2}{*}{$P$} \\
\hline & Ausente & Ocasional & Frequente & & \\
\hline Dorme pouco & $64,6(12,0)$ & $66,9(11,8)$ & $68,7(5,7)$ & 0,64 & 0,52 \\
\hline Desperta & $64,8(12,0)$ & $64,2(10,8)$ & $69,5(10,6)$ & 1,30 & 0,27 \\
\hline Pesadelo & $62,8(11,3)$ & $68,6(11,1)$ & $72, I(9,1) \#$ & 4,06 & 0,02 \\
\hline Fala, chora & $62,9(11,7)$ & $66,3(11,6)$ & 71,3 (8,4) \# & 3,41 & 0,03 \\
\hline
\end{tabular}

Na Tabela 3, encontra-se a comparação entre os escores T médios obtidos no Perfil Externalizante por crianças $(\mathrm{N}=83)$ de acordo com a frequência do problema de sono. Os escores no perfil externalizante de crianças com problemas frequentes para dormir, que resistem a ir para a cama, têm pesadelos ou que falam/choram durante o sono são significativamente mais altos do que os escores encontrados em crianças cujas mães não referiram essas dificuldades com o sono.

Do total de crianças participantes, $71 \%(n=59)$ tinham problemas externalizantes. Dessas 59 crianças, 35,6\% $(n=21)$ tinham problemas com o sono. Já das 24 crianças sem problemas externalizantes somente $3(12,5 \%)$ tinham problemas com o sono. A estimativa da razão de prevalência demonstrou que crianças com problemas de sono têm risco de $40 \%$ a mais para problemas externalizantes em comparação com as que não apresentam dificuldades com o sono ( $R P=1,4$; IC 95\% =1,07-1,73).

\section{Discussão}

Os resultados deste estudo sugerem que aproximadamente um terço das crianças participantes foi classificado como clínico para problemas de sono. Este dado pode indicar que problemas de sono acometem um número considerável de crianças em idade pré-escolar, além de ir ao encontro de estudos anteriores (Owens \& Mindell, 2011; Pires et al., 2012) que apontam que $25 \%$ a $40 \%$ da população infantil apresentam problemas de sono.

Dentre os problemas, foi observado em alta frequência o fato de a criança não querer dormir sozinha, apresentar problemas no momento de dormir, resistir a ir para a cama e despertar várias vezes durante a noite. Tais manifestações são características do diagnóstico de Insônia Comportamental Infantil. De fato, a insônia é um dos problemas de sono mais frequentes entre as crianças, o que já foi apontado por vários 
autores (Owens \& Mindell, 2011; Tikotzky \& Sadeh, 2010). Por meio dos itens do CBCL que contemplam os problemas de sono, é possível identificar problemas relacionados ao início e à manutenção do sono de forma satisfatória. Além de características da insônia, o instrumento também contempla duas questões referentes às parassonias, como "pesadelos" e "falar e chorar enquanto dorme", que foram menos frequentemente relatadas pelas mães na presente amostra.

A resistência a ir para a cama, característica da insônia do tipo de estabelecimento de limites, pode ser causada por vários fatores, entre eles, estilo parental permissivo, estilos de disciplina inconsistente entre os pais, bem como expectativas irrealistas por parte dos cuidadores (Mindell et al., 2006; Owens \& Mindell, 2011). Esses fatores devem ser identificados, pois podem estar mantendo os problemas de sono. A prática de dormir com os pais também foi observada em outros estudos (Sadeh, Tikotzky, \& Scher, 2010; Sourander, 2001) que sugerem a associação desta prática à menor qualidade de sono, uma vez que a criança vai para a cama mais tarde e desperta muitas vezes durante a noite em razão da partilha da cama e/ou do quarto.

Houve um número elevado de participantes que apresentou escores clínicos tanto no perfil internalizante quanto no externalizante. Por se tratar de uma amostra clínica, era esperada a ocorrência de problemas de comportamento de ambos os tipos, uma vez que tais comportamentos estão presentes em comorbidades psiquiátricas e comportamentais e que a etiologia desses problemas pode ser similar. A co-ocorrência de problemas internalizantes e externalizantes foi observada também no estudo de Borsa, Souza e Bandeira (2011), que aponta que uma mesma criança pode ser classificada como clínica em diferentes escalas e problemas de comportamento. Desta forma, pode-se inferir que o participante pode apresentar problemas internalizantes em determinados momentos e externalizantes em outros, dependendo do contexto ambiental.

Os resultados do presente estudo apresentaram uma possível associação entre problemas de sono e de comportamento. A comparação entre a frequência de problemas de sono apontou que os escores internalizantes e externalizantes foram mais altos nas crianças que apresentavam alguns problemas de sono do que nas crianças que não apresentavam. Esse dado sugere que crianças com problemas de sono têm maiores chances de apresentar pontuações maiores nas síndromes que compõem o perfil internalizante e externalizante do instrumento $C B C L$ pré-escolar. Os valores encontrados na análise de prevalência indicaram associação entre dificuldades com o sono e problemas internalizantes e externalizantes na presente amostra. Tais resultados são consistentes com a crescente evidência de que perturbações no sono podem ser prejudiciais ao comportamento de crianças. Vários estudos apontaram associação entre problemas de sono e problemas no perfil internalizante e/ou externalizante (Gregory et al., 2008; Hall et al., 2007; Scher et al., 2005; Sourander, 2001; Shang et al., 2006; Sivertsen et al., 2015; Stein et al., 2001; Storch et al., 2008).

Entretanto, diferentemente dos estudos citados, este estudo trata de uma população de crianças cujas mães que buscaram a clínica-escola por seus filhos apresentarem proble- 
mas comportamentais. Desta forma, chama-se a atenção ao fato de que problemas de sono devem ser investigados em crianças que apresentam problemas de comportamento.

Associações entre problemas de sono e de comportamento são provavelmente bidirecionais. Por um lado, os problemas de sono ou sono insuficiente exacerbam as dificuldades emocionais e comportamentais. Sobre este aspecto, há possíveis explicações pelas quais a interrupção de sono pode resultar em déficits durante o dia, indicando que a má qualidade de sono pode afetar a função do córtex pré-frontal, que regula muitos dos comportamentos que são sensíveis à interrupção do sono (Gregory \& Sadeh, 2012). A curta duração de sono pode influenciar na regulação emocional e aumentar a irritabilidade, que são características tanto de problemas de internalização quanto de externalização. Por outro lado, os problemas de comportamento podem comprometer os padrões de sono, tanto que as dificuldades de sono são apontadas como sintomas de uma variedade de diagnósticos, como ansiedade, depressão etc. (Gregory \& Sadeh, 2012).

Ao se tratar de crianças pequenas, fatores ambientais têm importante papel na relação entre sono e comportamento. Por exemplo, o estudo de Gregory, Eley, O'Connor, e Plomin, (2004) apontou que relatos de dificuldades com o sono e problemas internalizantes e externalizantes em crianças com 3 anos não foram relacionados com fatores genéticos, ao passo que fatores ambientais foram consistentemente associados tanto a problemas de sono quanto a problemas emocionais (internalizantes) e comportamentais (externalizantes).

Sivertsen et al. (2015) e Gregory et al. (2004) indicam que fatores familiares como desorganização familiar e depressão materna podem estar associados a problemas de sono e problemas internalizantes em crianças. Já a falta de rotina e de limites pode estar associada tanto a problemas internalizantes quanto a externalizantes. Os autores (Sivertsen et al., 2015) apontam ainda que a instabilidade conjugal entre os cuidadores possui associação com dificuldades com o sono e também com problemas internalizantes e externalizantes em crianças. O papel dos pais no desenvolvimento de problemas comportamentais e de sono já vem sendo evidenciado (Sadeh et al., 2010; Tikotzky \& Scher, 2010). Sivertsen et al. (2015) indicam ainda que problemas na relação pais-filhos estão associados a posteriores distúrbios de sono e a dificuldades comportamentais e emocionais.

Os problemas de sono podem persistir até a vida adulta e ocasionar prejuízos emocionais e comportamentais (Gregory et al., 2008; Tikotzky \& Sadeh, 2010). Esse fator é preocupante para o desenvolvimento infantil, uma vez que o sono é responsável por aproximadamente $40 \%$ do dia típico de uma criança. Desta forma, quando as crianças não dormem o suficiente, aspectos de seu desenvolvimento físico, emocional, cognitivo, comportamental e social podem ser afetados negativamente (Gregory \& Sadeh, 2012; Mindell et al., 2006).

Considerando o impacto do sono na qualidade de vida da criança e que muitas vezes os comportamentos dos pais e/ou cuidadores são responsáveis pelos comporta- 
mentos inadequados da criança no momento de dormir e ao despertar durante a noite, Mindell et al. (2006) apontam a importância de se tratar da problemática a partir de um treino terapêutico, de forma que os pais se tornem agentes ativos na mudança de comportamento de suas crianças. Esse tratamento visa modificar os comportamentos dos pais diante das respostas inadequadas da criança em relação ao sono, a fim de que os pais compreendam a origem dos comportamentos de seus filhos, para que, a partir da modificação de seus comportamentos, aconteça a modificação dos comportamentos da criança. Os autores pontuam que as intervenções para problemas de sono por meio de orientações para pais são efetivas, não só para a melhora do sono como também para problemas de comportamento que podem ser decorrentes da pouca qualidade de sono.

Associações entre sono e comportamento são complexas e provavelmente bidirecionais. Este estudo corrobora estudos anteriores que apontam uma associação entre problemas de sono e problemas de comportamento. Contudo, a presente amostra foi constituída de crianças cujas mães procuraram a clínica-escola em razão de a criança apresentar alguma queixa comportamental. O fato de não haver um grupo de controle para comparações com crianças que não apresentassem problemas de comportamento compromete a generalização dos dados, apresentando-se como uma limitação do atual estudo. Para conclusões gerais, são necessárias pesquisas que avaliem o sono em larga escala e experimentos controlados que estabeleçam os efeitos das variações de sono sobre as dificuldades emocionais e comportamentais da criança.

São importantes, também, estudos adicionais que avaliem o sono e o comportamento das crianças por meio de outros informantes, como pais, cuidadores de creche ou professores da pré-escola. A inclusão de múltiplos informantes possibilita melhor compreensão do funcionamento da criança, uma vez que é possível identificar o comportamento da criança em diferentes ambientes e interações.

Para melhor compreensão da relação entre sono e comportamento, pesquisas futuras são importantes para que sejam exploradas as características e a saúde mental dos pais, a rotina no ambiente familiar, a relação parental, o relacionamento conjugal e suas relações com problemas de sono infantil e problemas de ordem externalizante e internalizante. Explorar os fatores que estão associados à dificuldade de sono da criança é fundamental para o planejamento de intervenções efetivas (Gregory \& Sadeh, 2012).

Além disso, a presente pesquisa avaliou o sono por meio de itens que compõem a subescala Problemas de Sono do CBCL. A utilização de outros instrumentos para avaliar problemas e hábitos de sono na criança, bem como a utilização combinada de medidas objetivas de sono como a actigrafia, seria útil para maior detalhamento das características do sono nesta população.

Concluindo, o presente estudo demonstrou que problemas de sono são frequentes em crianças que foram levadas por suas famílias a uma clínica-escola para avaliação ou tratamento de problemas comportamentais, e que estão associados a problemas de comportamento internalizantes e externalizantes. Contudo, o sono e o comportamen- 
to das crianças foram avaliados por meio do CBCL pré-escolar e, apesar de esta versão ser bastante utilizada no país, suas normas ainda não foram validadas. Espera-se que este trabalho possa estimular a produção de pesquisas nacionais sobre o tema, servindo como incentivo para investigações de problemas de sono em crianças com problemas de comportamento e para o desenvolvimento de intervenções comportamentais que visem ao manejo da insônia na infância.

\section{Referências}

Achenbach, T. M., \& Rescorla, L. A. (2000). Manual for ASEBA preschool forms \& profiles. Burlington: University of Vermont, Research Center for Children, Youth, \& Families.

American Academy of Sleep Medicine. (2005). International classification of sleep disorders: diagnostic and coding manual, second ed. Westchester: American Academy of Sleep Medicine.

Borsa, J. C., Souza, D. S., \& Bandeira, D. R. (2011). Prevalência dos problemas de comportamento em uma amostra de crianças do Rio Grande do Sul. Psicologia: Teoria e Prática, 13(2), 15-29.

Gregory, A. M., Eley, T. C., O'Connor, T. G., \& Plomin, R. (2004). Etiologies of associations between childhood sleep and behavioral problems in a large twin sample. Journal of the American of Academy of Child and Adolescent Psychiatry, 43(6), 744-751. DOI: 10.1097/01.chi/0000122798.47863.a5.

Gregory, A. M., Ende, J. V., Willis, T. A., \& Verhulst, F. C. (2008). Parent-reported sleep problems during development and self-reported anxiety/depression, attention problems, and aggressive behavior later in life. Archive of Pediatrics \& Adolescent Medicine, 162(4), 330-335. DOI: 10.1001/archpedi.162.4.330.

Gregory, A. M., \& Sadeh, A. (2012). Sleep, emotional and behavioral difficulties in children and adolescents. Sleep Medicine Reviews, 16(2), 129-136. DOI: 10.1016/j. smrv.2011.03.007.

Hall, W. A., Scher, A., Zaidman-Zait, A., Espezel, H., \& Warnock, F. (2011). A community-based study of sleep and behaviour problems in 12-to-36 month old children. Child Care, Health, and Development, 38(3), 379-389. DOI: 10.1111/j.1365-2214.2011.01252.x.

Hall, W. A., Zubrick, S. R., Silburn, S. R., Parsons, D. E., \& Kurinczuk, J. J. (2007). A model for preditcting behavioural sleep problems in a random sample of australian pre-schoolers. Infant and Child Development, 16(5), 509-523. DOI: 10.1002/icd.527.

Hiscock, H., Bayer, J. K., Hampton, A., Ukoumunne, O. C., \& Wake, M. (2008). Long-term mother and child mental health effects of a population - based infant sleep intervention:cluster-randomized, controlled trial. Pediatrics, 122(3), e621-e627. 
Mindell, J. A., Kuhn, B., Lewin, D. S., Meltzer, L. J., \& Sadeh, A. (2006). Behavioral treatment of bedtime problems and night wakings in infants and young children. Sleep, 29(10), 1263-1276.

Owens, J. A., \& Mindell, J. A. (2011). Pediatric insomnia. Pediatric Clinics of North America, 58(3), 555-569. DOI: 10.1016/j.pcl.2011.03.011.

Pires, M. L. N., Vilela, C. B., \& Câmara, R. L. (2012). Desenvolvimento de uma medida de hábitos de sono e aspectos da prevalência de problemas comportamentais de sono na infância: uma contribuição. In D. P. S. A. Ribeiro, H. R. Rosa, N. S. Filho (Eds.). Processos clínicos e saúde mental (pp. 167-192). São Paulo: Vetor.

Sadeh, A., Tikotzky, L., \& Scher, A. (2010). Parenting and infant sleep. Sleep Medicine Reviews, 14(2), 89-96. DOI: 10.1016/j.smrv.2009.05.003.

Scher, A., Zukerman, S., \& Epstein, R. (2005). Persistent night waking and settling difficulties across the first year: early precursors of later behavioural problems? Journal of Reproductive and Infant Psychology, 23(1), 77-88. DOI: 10.1080/ 02646830512331330929.

Shang, C. H., Gau, S. S. F., \& Soong, W. T. (2006). Association between childhood sleep problems and perinatal factors, parental mental distress and behavioral problems. Journal of Sleep Research, 15(1), 63-73. DOI: 10.1111/j.1365-2869. 2006.00492.x.

Sivertsen, B., Harvey, A. G., Reichborn-Kjennerud, T., Torgersen, L., Ystrom, E., \& Hysing, M. (2015). Later emotional and behavioral problems associated with sleep problems in toddlers: a longitudinal study. The Jounal of the American Medical Association Pediatrics, 169(6), 575-582. DOI: 10.1001/jamapediatrics. 2015.0187.

Sourander, A. (2001). Emotional and behavioural problems in a sample of finnish three-year-olds. European Child \& Adolescent Psychiatry, 10(2), 98-104. DOI: $10.1007 / 5007870170032$.

Stein, M. A., Mendelsohn, J., Obermeyer, W. H., Amromin, J., \& Benca, R. (2001). Sleep and behavior problems in scholl-aged children. Pediatrics, 107(4), E60.

Storch, E. A., Murphy, T. K., Lack, C. W., Geffken, G. R., Jacob, M. L., \& Goodman, W. K. (2008). Sleep-related problems in pediatric obsessive-compulsive disorder. Journal of Anxiety Disorders, 22(5), 877-888. DOI: 10.1016/j.janxdis.2007.09.003.

Tikotzky, L., \& Sadeh, A. (2010). The role of cognitive-behavioral therapy in behavioral childhood insomnia. Sleep Medicine, 11(7), 686-691. DOI: 10.1016/j.sleep.2009.11.017. 